\title{
The Impact of COVID-19 Pandemic on Surgical Primary Retinal Detachments
}

\author{
André Ferreira $^{a, b}$ Miguel Afonso $^{a}$ Nisa Silva $^{a}$ Angelina Meireles ${ }^{a, c}$ \\ aService of Ophthalmology, Centro Hospitalar Universitário do Porto, Porto, Portugal; 'bunit of Anatomy, \\ Department of Biomedicine, Faculty of Medicine of University of Porto, Porto, Portugal; ' Department of \\ Ophthalmology, Instituto de Ciências Biomédicas Abel Salazar, University of Porto, Porto, Portugal
}

\section{Keywords}

Coronavirus disease 19 . Primary retinal detachment .

Vitrectomy

\begin{abstract}
Purpose: This study aimed to describe the impact of COVID-19 pandemic on the presentation characteristics, timings, and surgical decisions for primary rhegmatogenous retinal detachment (RRD). Methods: A historical cohort including all patients who presented to the Surgical Retina Section at Centro Hospitalar Universitário do Porto (CHUPorto), over a 2-year period, was recruited and divided into 2 groups: pre-COVID and COVID groups. The onset of the COVID-19 pandemic was recorded as starting on March 18, 2020, the same day the first pandemic-related lockdown came to effect in Portugal. Results: This study enrolled 449 eyes of 443 patients: 272 in the pre-COVID group and 177 in the COVID one. Of the patients, $63.6 \%$ were male, and the mean \pm SD age was $63.0 \pm 13.2$ years (range $13-92$ years). Of the eyes, $55.5 \%(n=151)$ presented with macular detachment in the pre-COVID group compared with $66.9 \%(n=119)$ in the COVID group (odds ratio [OR] 1.62; 95\% confidence interval $[\mathrm{Cl}]: 1.09-3.86 ; p=0.016)$. The time from symptom
\end{abstract}

karger@karger.com

(c) 2021 S. Karger AG, Basel

www.karger.com/oph

Karger! onset to hospital admission $(p=0.021)$ and from admission to surgery $(p<0.001)$ was longer in the COVID era. In the COVID period, silicone oil (OR 2.03, 95\% Cl: 1.09-3.79, $p=0.025$ ) and C3F8 gas (OR 2.42, 95\% Cl: 1.57-3.71, $p<0.001$ ) were used more often. No differences in anatomical success or final visual acuity were found. Conclusions: The lockdown due to COVID pandemic affected the epidemiology of RRD. The services must adapt to the novel reality and produce backup plans for similar events. Despite the contingencies, the final results were not different between groups.

(c) 2021 S. Karger AG, Basel

\section{Introduction}

In December 2019, an outbreak of a novel coronavirus disease (COVID-19) emerged in Wuhan [1]. The infection rapidly spread worldwide, causing the WHO to declare it a pandemic on March 11 [2].

Aiming to concentrate efforts to deal with the infected patients and to mitigate the nosocomial spread of the virus, healthcare systems had to rapidly reshape their framework [3]. In Portugal, like in most countries, the policies led to the curtailment of physician office hours,

Correspondence to:

André Ferreira, andre.ferreira@live.com.pt 
restriction of elective procedures, and postponement of elective health visits, reducing patients' access to health professionals. At the same time, patients' concerns for exposure, coupled with distancing measures, may have led patients to stay at home even when an acute medical condition develops.

Rhegmatogenous retinal detachment (RRD) is an urgent ophthalmic condition that may result in significant vision loss, particularly when the macula is affected. Delay in presentation and management not only increases the probability of macula involvement but is also associated with worse final visual acuity and surgical outcomes, including the need for several and more complex surgeries [4-7]. This study sought to compare the epidemiology and outcomes of surgical RRD between 2 consecutive years, pandemic year and the previous one.

\section{Methods}

In this retrospective cohort, all consecutive patients who presented to the Surgical Retina Unit at Centro Hospitalar Universitário do Porto (CHUPorto), over a 2-year period, were recruited and divided into 2 groups: pre-COVID group, from March 18, 2019, to March 17, 2020, and COVID group, from March 18, 2020, to March 17, 2021. The onset of the COVID-19 pandemic was recorded as starting on March 18, 2020, the same day the first pandemic-related lockdown came to effect in Portugal [8]. Patients were identified by the ICD-9 and ICD-10 codes. The research adhered to the principles of the Declaration of Helsinki and its latest amendment (Brazil, 2013). All patients provided informed consent for treatment, and the study protocol complies with the requirements of the institute's committee on human research.

Patients were only included if they presented between the specified dates with a primary RRD and had at least 6 weeks of followup after pars plana vitrectomy (PPV), scleral buckle (SB), or combined PPV and SB. Patients were excluded if the affected eye was treated with laser retinopexy for a retinal tear only, or if the affected eye had pre-existing maculopathy, retinopathy, amblyopia, or prior history of serous, tractional, or RRD in the affected eye.

\section{Surgical Repair}

SBs were secured to the sclera using partial thickness bites of 5-0 mersilene sutures. The type of buckle, the decision between encircling versus segmental buckles, and the buckle height were done at surgeon's discretion.

PPV procedures were performed using a 23-gauge transconjunctival system, with $360^{\circ}$ peripheral vitreous shave visualized by scleral indentation. Laser retinopexy was preferred to treat most retinal pathologic features. However, supplementary cryopexy was applied as needed in patients with small, peripheral, anterior retinal breaks. Subretinal fluid generally was drained through the break responsible for the retinal detachment. The use of heavy liquid or posterior retinotomy was at the surgeon's discretion. A complete air-fluid exchange was performed, and an isoexpansile SF6 or C3F8 gas or silicone oil was injected. SB or combined cata- ract extraction was at the surgeon's discretion. Patients were placed face down immediately after PPV, except in macula-on patients with no SRF close to the posterior pole at the end of surgery, in which case they were positioned according to the location of the retinal break. The therapeutic regimen after surgery has been described elsewhere [9].

\section{Outcomes}

Baseline characteristics (age, gender, laterality, referral, and time from symptom onset to hospital presentation and to surgery), type of treatment, RD characteristics at presentation (status of macula, lens status, and visual acuity), and visual acuity and retinal status after surgery were collected. For statistical purposes, "counting fingers" was classified as 0.01 , "hand movement" as 0.005 , and "light perception" as 0.0005 [10]. Corrected distance visual acuity (CDVA) registered in decimals was converted to the logarithm of the minimum angle of resolution equivalent [11]. Primary anatomical success was considered retinal reattachment at 12 weeks after 1 procedure. Final visual acuity was assessed at 12 weeks after surgery.

The primary outcome measures were the status of the macula, CDVA on presentation, and time from admission to surgery between the pre-COVID and the COVID pandemic cohorts. A subanalysis by age was performed using 50 years as the cutoff. Secondary outcomes were the duration of symptoms, type of tamponade, primary anatomical success, and final CDVA between the 2 cohorts.

\section{Statistical Analyses}

All statistical analyses were performed using Stata software (StataCorp. 2015, Stata Statistical Software: Release 14; StataCorp LP, College Station, TX, USA). Shapiro-Wilk, KolmogorovSmirnov test, and normal probability plots were used to confirm the normal distribution of the data. Categorical variables were described through absolute and relative frequencies, and continuous variables were described through means and standard deviations, or medians and ranges for variables with a skewed distribution. Parametric or nonparametric tests were used according to the normality of data. $\chi^{2}$ or Fisher's exact tests were performed for categorical variable comparison. Bonferroni correction was used for subanalysis of the categorical variable. Statistical significance was defined as $p<0.05$.

\section{Results}

This study enrolled 449 eyes of 443 patients: 272 in the pre-COVID group and 177 in the COVID one, representing a reduction of $35 \%$ in the number of cases. Table 1 presents the demographics and baseline characteristics of the patients enrolled. Of the patients, $63.6 \%$ were male, and the mean \pm SD age was $63.0 \pm 13.2$ years (range $13-92$ years). Overall, $74.83 \%$ of visits were referrals. No differences were found regarding age, gender, or referral between groups.

The majority of the eyes $(59.0 \%, n=259)$ were phakic with no differences found between periods. One-hundred and fifty-one eyes $(55.5 \%)$ presented with macular de- 
Table 1. Characteristics of population at hospital admission

\begin{tabular}{|c|c|c|c|}
\hline & $\begin{array}{l}\text { Pre-COVID } \\
(n=272)\end{array}$ & $\begin{array}{l}\text { COVID } \\
(n=177)\end{array}$ & $p$ value \\
\hline Right eye, $n(\%)$ & $151(55.5)$ & $92(52.0)$ & 0.46 \\
\hline Male gender, $n(\%)$ & $175(64.3)$ & $111(62.7)$ & 0.73 \\
\hline Age, mean $\pm S D$ & $63.54 \pm 13.44$ & $62.23 \pm 12.91$ & 0.31 \\
\hline Referral, $n(\%)$ & $203(75.7)$ & $130(73.4)$ & 0.58 \\
\hline \multicolumn{4}{|l|}{ Lens status, $n(\%)$} \\
\hline Phakia & $152(57.1)$ & $107(61.8)$ & \\
\hline Pseudophakia & $110(41.4)$ & $66(38.2)$ & 0.20 \\
\hline Aphakia & $4(1.5)$ & $0(0.0)$ & \\
\hline Visual acuity, mean $\pm S D$, logMAR & $1.33 \pm 0.92$ & $1.45 \pm 0.87$ & 0.19 \\
\hline \multicolumn{4}{|l|}{ Macula status, $n(\%)$} \\
\hline Off & $151(55.5)$ & $118(67.0)$ & \\
\hline On & $71(26.1)$ & $45(25.6)$ & 0.003 \\
\hline On-off & $50(18.4)$ & $13(7.4)$ & \\
\hline Time from symptom onset to hospital admission, median (IQR), days & $7(3-10)$ & $7(3-15)$ & 0.021 \\
\hline Time from hospital admission to surgery, median (IQR), days & $0(0-2)$ & $2(1-4)$ & $<0.001$ \\
\hline
\end{tabular}

Table 2. Surgical outcomes and postoperative visual acuity

\begin{tabular}{|c|c|c|c|}
\hline & $\begin{array}{l}\text { Pre-COVID } \\
(n=272)\end{array}$ & $\begin{array}{l}\text { COVID } \\
(n=177)\end{array}$ & $p$ value \\
\hline Scleral buckle, $n$ (\%) & $8(2.9)$ & $3(1.7)$ & 0.40 \\
\hline \multicolumn{4}{|l|}{ Tamponade, $n(\%)$} \\
\hline SF6 & $3(37.5)$ & $0(0)$ & \multirow{5}{*}{0.10} \\
\hline C3F8 & $0(0)$ & $0(0)$ & \\
\hline Air & $0(0)$ & $1(33.3)$ & \\
\hline BSS & $0(0)$ & $1(33.3)$ & \\
\hline None & $5(62.5)$ & $1(33.3)$ & \\
\hline Pars plana vitrectomy, $n$ (\%) & $264(97.1)$ & $174(98.3)$ & 0.40 \\
\hline \multicolumn{4}{|l|}{ Tamponade, $n(\%)$} \\
\hline SF6 & $169(64.0)$ & $77(44.2)$ & \multirow{4}{*}{$<0.001$} \\
\hline C3F8 & $66(25.0)$ & $71(40.8)$ & \\
\hline Air & $9(3.4)$ & $2(1.1)$ & \\
\hline Silicone oil & $20(7.6)$ & $24(13.8)$ & \\
\hline Primary anatomical success, $n$ (\%) & $225(82.7)$ & $152(85.9)$ & 0.37 \\
\hline Primary anatomical success, ${ }^{*} n(\%)$ & $210(83.3)$ & $132(86.3)$ & 0.43 \\
\hline Postop VA, mean $\pm S D$, logMAR & $0.63 \pm 0.63$ & $0.68 \pm 0.64$ & 0.53 \\
\hline
\end{tabular}

* After exclusion of patients with silicone oil tamponade. tachment in the pre-COVID group compared to $66.9 \%$ $(n=119)$ in the COVID group (odds ratio [OR] 1.62; 95\% confidence interval [CI]: 1.09-3.86; $p=0.016)$. We performed a subanalysis of macular status according to age subgroups using 50 years as the cutoff. In the control year, no differences were found. In the COVID year, older patients were less likely to present with macula attached (OR 0.43, 95\% CI: 0.19-1.00, $p=0.050$ ).

The time from symptom onset to hospital presentation was longer in the COVID era $(p=0.021)$. As well, the time from hospital presentation to surgery was also longer during the pandemic year $(p<0.001)$. A subanalysis of the patients that were not submitted to surgery in the presentation day $(n=263)$ also revealed a higher waiting time until surgery in the COVID year (median [IQR]: 2 [1-3] vs. 3 [2-4.5] days, $p<0.001$ ).

Table 2 describes the surgical procedures and postoperative visual acuity. PPV was performed in $97.6 \%$ of cases, and the overall anatomical success of interventions (PPV and SB) was $84.2 \%$, no differences between periods. 
In the COVID year, silicone oil (OR 2.03, 95\% CI: 1.09$3.79, p=0.025$ ) and C3F8 gas (OR 2.42, 95\% CI: $1.57-$ $3.71, p<0.001)$ were used more often. No differences were found regarding final visual acuity.

\section{Discussion}

The COVID-19 pandemic has paralyzed the healthcare systems globally, either with the burden imposed by the infected patients or with the lockdown and restriction policies that delayed the timely observation of potentially life-threatening conditions. Several authors have described the impact of this pandemic in the ophthalmology practice [12-15]. We selected RRD as an index disease for study because its symptoms warrant urgent evaluation, and we aimed to assess the impact of the pandemic on the patients' attitudes toward self-care and on hospital's management of acute non-COVID conditions. In this study, we reviewed all consecutive patients admitted to our service with the diagnosis of primary RRD submitted to surgery in the last 2 years, the pre-COVID and the COVID years. To avoid bias due to seasonal variations in the incidence of RRD [16], we chose to compare 2 consecutive years. During the pandemic year, there was a $35 \%$ reduction in the number of patients with RRD, the patients presented more frequently with macular detachment, mostly the older ones, and waited more time since symptom onset until hospital presentation and since hospital presentation to surgery. Moreover, the resource of silicone oil and C3F8 gas tamponade was also higher during the COVID-19 year. To our best knowledge, this is the largest cohort of surgical RRD patients comparing the pre-COVID and COVID years.

The Surgical Retinal Unit of our service has the highest number of patients with RRD in the country, receiving referrals from all regions in the north of Portugal. Before the pandemic, all patients diagnosed with an RRD at our institution were observed by a retinal surgeon within 24 $\mathrm{h}$ after presentation. If the macula was attached, the surgery was scheduled for the same day or the next; if the macula was affected, the surgery would happen within 72 h. To face the adversities imposed by the COVID pandemic, a specific protocol for this disease was designed. The senior author of this study (A.M.) was on-call from 9 a.m. to 11 p.m. (or 8 p.m., when the nightshift is assured by the other tertiary hospital center of Porto, shifting every 2 weeks), 7 days a week, to give specific instructions including the priority for patients presenting in the emergency room with RRD. When on holidays, this task was attributed to another senior member of the unit. These instructions meant to facilitate the route from hospital presentation to surgery and included the foreseen day and hour of surgery, the day for SARS-CoV-2 testing, and other lab exams as needed. Even with the adaptions adopted, patients waited more time since hospital presentation until surgery due to the need for SARS-CoV-2 testing and the less availability of operating rooms.

In both periods, about three-quarters of our patients were referred. During the pandemic year, some of the referring institutions opted to contact directly the service and were given a specific date for the patient to come for assessment by our Surgical Retinal Unit in the morning with a reserved space in that afternoon surgical schedule should he had indication for surgery. We performed a subanalysis of the waiting time to surgery excluding those undergoing surgery in the presentation day to evaluate the real effect of the pandemic and to avoid this potential bias. The waiting time until surgery was longer for the COVID-19 period in both analyses. Despite these contingencies, the postoperative visual acuity and anatomical success were similar for both periods. Albeit nonsignificant, the primary anatomical success was superior in the COVID-19 year, even after exclusion of patients with silicone oil tamponade. This fact might reflect some intraoperative extra measures not explored in the study such as more extensive retinal laser or cryotherapy. Moreover, as patients spent more time at home due to lockdown, the explanation might involve a higher compliance to postoperative positioning and more aid from householders in the diary task with less efforts performed by the patients. Although the COVID pandemic might have influenced some intraoperative decisions, PPV was considered the treatment of choice in both periods. The demand for anesthesiologists to perform other tasks during the COVID period, namely, in the intensive care units, did not influence our practice or surgical techniques as all ophthalmological surgeries were performed with anesthesiology support. Other external factors, including material availability or surgeons' absence due to own or householders COVID infection, did not affect either the standard of care.

Similar to other reports $[14,15,17-19]$, we found a reduction of almost 100 cases (35\%) in the total number of RRD during the pandemic year. We also report a delayed presentation and a higher prevalence of macular detachment in the pandemic year, mostly in older patients, in agreement with other authors [12, 13, 17-20]. In this specific scenario, the real reasons for this delay were not formally documented in patients' charts, but similar to Arjmand et al. [12], we found some anecdotal evidence of 
later presentations due to fear of contracting COVID-19. As Patel et al. [20] advocate, the social perception of lower morbidity and mortality of COVID-19 for the younger population might have resulted in less reluctancy for seeking healthcare evaluation. Beyond younger age, Patel et al. [20] also reported that patient established status was a predictor of macula-on RRD at presentation. In the pandemic setting, patients with an established follow-up might face fewer logistical barriers to evaluation and might be more able to consider a risk-to-benefit analysis in case of new symptom onset [20]. In fact, an association between worse patient education and later presentation has been established, justified by the lower awareness of symptoms and potential morbidity [21,22].

Proliferative vitreoretinopathy (PVR) is associated with poor prognosis after RRD repair, including treatment failure [23], with its prevalence being higher in developing countries with suboptimal access to healthcare [24]. Arjmand et al. [12] found no differences at baseline in the prevalence of PVR, whereas other authors $[17,20]$ reported an increased number of cases with this feature at admission, associating its presence with a delayed presentation, an increased need for multiple and complex surgery, and a higher chance of failure. In our study, this feature was not assessed as it was not consistently reported in the charts. In the pandemic year, the resource to silicone oil and C3F8 gas was higher in our cohort which can be taken as a surrogate of the increased severity of cases. This fact was also supported by others [17]. This fact might also reveal some reluctancy by the surgeons, aiming to avoid at all cost relapses and further surgeries.

This is a retrospective study having the inherent bias to this design. As already mentioned, PVR is one important aspect of the RRD disease spectrum and was not assessed by us. The anatomic success and final CDVA were assessed using the 3-month mark as the last follow-up visit. The onset of symptoms was considered as a class without discerning floaters and flashes from visual field defect and might have been influenced by the inherent subjectivity. However, this fact is valid for both periods, and a differential recall bias seems unlikely.

\section{Conclusion}

Our findings and the available evidence on this subject point out that the COVID-19 pandemic affected the epidemiology of acute RRD, with a shift toward more macula detached at presentation and a longer waiting time before seeking care. The approach was also adjusted with the silicone oil being more often used. The services must adapt to the novel reality and produce backup plans for similar events. Public instructions on the most important signs and symptoms of RRD are needed, aiming to anticipate the evaluation of suspect or pre-rhegmatogenous lesions and, in some circumstances, even avoid RRD. Policies for a coordinated national response of the Ophthalmology Departments and a countrywide protocol would help to face similar scenarios with a homogenous response throughout the services. Long-term follow-up results are warranted to evaluate the socioeconomic burden in the visual healthcare imposed by this pandemic. At the time of this writing, we were facing the third wave of COVID pandemic, and we still do not know how much more time this scenario will last, emphasizing the importance of these results to future decisions.

\section{Acknowledgements}

We are grateful to Mrs Lucinda Silva for her technical support.

\section{Statement of Ethics}

The study was conducted under the tenets of the National Council of Ethics for the Life Sciences and the Declaration of Helsinki and its latest amendment (Brazil, 2013). Informed consent was waived by the Local Ethics Committee of all participating centers before study initiation.

\section{Conflict of Interest Statement}

The authors declare no conflicts of interest.

\section{Funding Sources}

There was no funding.

\section{Author Contributions}

A.M. and A.F. contributed to study design; A.F., M.A., and N.S. contributed to data acquisition; A.F. contributed to data analysis; all authors contributed to critical interpretation of data, manuscript drafting, and manuscript critical review.

\section{Data Availability Statement}

The datasets analyzed during the current study are available from the corresponding author A.F. upon reasonable request. 


\section{References}

1 Zhu N, Zhang D, Wang W, Li X, Yang B, Song $\mathrm{J}$, et al. A novel coronavirus from patients with pneumonia in China, 2019. N Engl J Med. 2020;382(8):727-33.

2 World Health Organization. Statement on the meeting of the International Health Regulations (2005) Emergency Committee regarding the outbreak of novel coronavirus (2019nCoV). 2020. Available from: https://www. who.int/news-room/detail/30-01-2020-statement-on-the-second-meeting-of-the-international-health-regulations-(2005)-emergency-committee-regarding-the-outbreakof-novel-coronavirus-(2019-ncov.

3 Arora VM, Chivu M, Schram A, Meltzer D. Implementing physical distancing in the hospital: a key strategy to prevent nosocomial transmission of COVID-19. J Hosp Med. 2020;15(5):290-1.

4 Rehman Siddiqui MA, Abdelkader E, Hammam T, Murdoch JR, Lois N. Socioeconomic status and delayed presentation in rhegmatogenous retinal detachment. Acta Ophthalmol. 2010;88(8):e352-3.

5 Tani P, Robertson DM, Langworthy A. Prognosis for central vision and anatomic reattachment in rhegmatogenous retinal detachment with macula detached. Am J Ophthalmol. 1981;92(5):611-20.

6 Greven MA, Leng T, Silva RA, Leung LB, Karth PA, Moshfeghi DM, et al. Reductions in final visual acuity occur even within the first 3 days after a macula-off retinal detachment. Br J Ophthalmol. 2019;103(10):1503-6.

7 van Bussel EM, van der Valk R, Bijlsma WR, La Heij EC. Impact of duration of macula-off retinal detachment on visual outcome: a systematic review and meta-analysis of literature. Retina. 2014;34(10):1917-25.

8 Silva N, Laiginhas R, Meireles A, Barbosa Breda J. Impact of the COVID-19 pandemic on ophthalmology residency training in Portugal. Acta Med Port. 2020;33(10):640-8.
9 Silva N, Ferreira A, Marques JH, Ferreira N, Correia N, Pessoa B, et al. Epiretinal membrane vitrectomy: outcomes with or without cataract surgery and a novel prognostic factor for cystoid macular edema. Graefes Arch Clin Exp Ophthalmol. 2021;259(7):1731-40.

10 Lange C, Feltgen N, Junker B, Schulze-Bonsel $\mathrm{K}$, Bach M. Resolving the clinical acuity categories "hand motion" and "counting fingers" using the freiburg visual acuity test (FrACT). Graefes Arch Clin Exp Ophthalmol. 2009; 247(1):137-42.

11 Holladay JT. Proper method for calculating average visual acuity. J Refract Surg. 1997; 13(4):388-91.

12 Arjmand P, Murtaza F, Eshtiaghi A, Popovic MM, Kertes PJ, Eng KT. Impact of the COVID-19 pandemic on characteristics of retinal detachments: the Canadian experience. Can J Ophthalmol. 2021;56(2):88-95.

13 Franzolin E, Longo R, Casati S, Ceruti P, Marchini G. Influence of the COVID-19 pandemic on admissions for retinal detachment in a tertiary eye emergency department. Clin Ophthalmol. 2021;15:2127-31.

14 Poyser A, Deol SS, Osman L, Sivagnanasithiyar T, Kuht HJ, Manrique R, et al. Impact of COVID-19 pandemic and lockdown on retinal detachments. Eye. 2021;35(8):2322-23.

15 Wickham L, Hay G, Hamilton R, Wooding J, Tossounis H, da Cruz L, et al. The impact of COVID policies on acute ophthalmology services-experiences from Moorfields Eye Hospital NHS Foundation Trust. Eye. 2020;34(7): 1189-92.

16 Kim DY, Hwang H, Kim JH, Moon BG, Hyung SM, Kim JY, et al. The association between the frequency of rhegmatogenous retinal detachment and atmospheric temperature. J Ophthalmol. 2020;2020:2103743.
17 Awad M, Poostchi A, Orr G, Kumudhan D, Zaman A, Wilde C. Delayed presentation and increased prevalence of proliferative vitreoretinopathy for primary rhegmatogenous retinal detachments presenting during the $\mathrm{CO}$ VID-19 pandemic lockdown. Eye. 2021; 35(4):1282-3.

18 Rohl A, Kalhorn A, Singh J, Mandava N. Decreased retinal detachments during a COVID-19 lockdown period in Colorado. Acta Ophthalmol. 2021;99(4):e618-9.

19 Shams F, El-Abiary M, Goudie C, Yorston D. Effects of lockdown on retinal detachment incidence in Scotland. Eye. 2021;35(4):1279-80.

20 Patel LG, Peck T, Starr MR, Ammar MJ, Khan MA, Yonekawa Y, et al. Clinical presentation of rhegmatogenous retinal detachment during the COVID-19 pandemic: a historical cohort study. Ophthalmology. 2021;128(5): 686-92.

21 Goezinne F, La Heij EC, Berendschot TT, Tahzib NG, Koetsier LS, Hoevenaars JG, et al. Patient ignorance is the main reason for treatment delay in primary rhegmatogenous retinal detachment in The Netherlands. Eye. 2009;23(6):1393-9.

22 Eijk ES, Busschbach JJ, Timman R, Monteban HC, Vissers JM, van Meurs JC. What made you wait so long? Delays in presentation of retinal detachment: knowledge is related to an attached macula. Acta Ophthalmol. 2016; 94(5):434-40.

23 Adelman RA, Parnes AJ, Michalewska Z, Ducournau D; European Vitreo-Retinal Society Retinal Detachment Study Group. Clinical variables associated with failure of retinal detachment repair: the European vitreo-retinal society retinal detachment study report number 4. Ophthalmology. 2014;121(9):1715-9.

24 Tseng W, Cortez RT, Ramirez G, Stinnett S, Jaffe GJ. Prevalence and risk factors for proliferative vitreoretinopathy in eyes with rhegmatogenous retinal detachment but no previous vitreoretinal surgery. Am J Ophthalmol. 2004;137(6):1105-15. 